A preliminary study of a cloth guiding mechanism for automatic sewing system.

\title{
Shigeru Inui
}

Department of Textile Science and Technology, Shinshu- University

\section{Tatsuro Yamada}

Seiren. Co. Ltd

\section{Yosuke Horiba}

Department of Textile Science and Technology, Shinshu- University

\section{Minoru Hashimoto}

Department of Textile Science and Technology, Shinshu- University 


\section{Abstruct}

Purpose - In the trend from mass production to mass customization, more flexible production systems are required. In clothing field, many studies about automatization of sewing processes have been done to product small amount of various kinds of products. Here, a versatile guiding mechanism of a cloth for automatic sewing system is proposed.

Methodology/Approach - Real sewing processes were referenced for the mechanism, and curved stitch is formed holding a point on a cloth. This mechanism consists of a solenoid for holding a cloth and a roller to prevent deformation of the cloth. When a cloth is sewn with the mechanism, the trajectory of the stitch is unstable because of anisotropy of a cloth. A precise trajectory was obtained by adding a device to control the pressure of the roller for holding a cloth and keeping a tension properly applied to a cloth.

Findings - It was found out that shearing property is the most related to the stability of sewing trajectory. If the tension for guidance applied to a cloth is constant, deformation of the cloth was observed and it was the cause of unstableness of sewing trajectory. By controlling the tension for guidance applied to a cloth properly according to the direction of the cloth, precise sewing trajectory was obtained.

Originality/value-

There have been some studies in which sewing conditions were dynamically controlled according to the mechanical properties of a cloth. To these studies, here it was proposed that sewing conditions were kept constant by controlling the guidance of a cloth according to its mechanical properties. 


\section{Introduction}

Nowadays, we can obtain clothing at a low price owing to mass production and mass consumption. On the other hand, many consumers are looking for unique clothing. Flexible manufacturing processes for clothing are required to respond to the demand of consumers at a reasonable cost. Development of automatic sewing system is required to realize the flexible manufacturing processes.

About two decades ago, a project of automatic sewing system of Ministry of International Trade and Industries had started in Japan. In the project, many instruments developed, such as a small sewing machine that was attached to a robot arm and could sew in 3D space, a robot could pick up a sheet of fabric from a pile or an instrument with many needles that could pick up a sheet of fabric and so on. Most of the instruments were designed for a single process, and they couldn't adapt to the wide variety of cloths. Because of this, most of the instruments were not introduced to manufactures.

Recently, there are some studies about automatic sewing. Stylios et al. presented a control system for sewing machine (Stylios and Sotomi, 1994). The system predicted foot pressure and disk pressure from material characteristics and sewing speed utilizing neural network and fuzzy model. Rocha et al. made a sewing machine instrumented with sensors on an experimental basis (Rocha et al., 1996). Rogale et al. took the same approach to develop a measuring system for sewing parameter acquisition (Rogale and Dragcevic, 1998). Carvalho et al. quantified the sewability of different materials and tried to control an overlock sewing machine(Carvalho and Ferreira, 2003). 
We focused on an automatic sewing system for sewing processes and aimed at a simple and versatile system. Some of sewing processes are straight stitch and others are curved one, and for curved stitch, guiding a cloth is very important. In sewing processes, operator of sewing machine guides a cloth to be fed constantly so that it is not necessary to change sewing conditions. Guide of a cloth in sewing processes have to be controlled according to the properties of the cloth, and operator of sewing machine actualizes unconsciously. As a first step, the purpose of this study is to guide a cloth to form fine curved stitch by considering characteristics of each cloth.

\section{A guiding mechanism.}

In the sewing processes of a shirt manufacturing company, it was observed that a skilled worker formed curved stitch rotating a cloth around a point at which he was holding a cloth with his fingers. With reference to the sewing processes, we constructed a mechanism that held a cloth with a solenoid at a point beside a differential feed cog. A cloth was rotated around the point and curved stitch was formed. The sewing machine utilized in this study was for domestic use, and the sewing speed for the experiment was about 600 stitches per minute. The mechanism with solenoid held a cloth only at a point, and the cloth was hardly applied excess force and was not deformed. A widely used bush type solenoid was utilized for the mechanism to hold a point on a cloth. When the solenoid was applied a voltage, an iron core of 3mm stroke stuck out and collided with a base. The vertical load applied by the iron core was enough to hold tightly the cloth. A driving circuit for the solenoid was assembled and interfaced with a PC.

When curved stitch was formed with the guiding mechanism, deformation of a cloth appeared in front of 
the holding point, and prevented an accurate control of a cloth. We decided to add a roller to the system that applied tension to the cloth to avoid deformation. The roller was made of polyurethane, width $10 \mathrm{~mm}$, diameter 30mm and notched on the surface. This type of roller was expected that the greater frictional force could be obtained compared to normal type of rollers. The solenoid was arranged at the position $70 \mathrm{~mm}$ left side of a sewing needle. The appearance of the cloth guiding mechanism with solenoid is shown in Figure1, and the arrangement of the sewing needle, the solenoid and the roller is shown in Figure 2. To avoid the deformation of a cloth and to enable sewing curved stitch around the holding point, a roller was added to the cloth guiding mechanism.

Taken in Figure 1

Taken in Figure 2

\section{Sewing Problems}

The pressure of the roller had to be controlled at a proper magnitude. The roller was pressed on a cloth and revolved to apply a tension to the cloth for avoiding deformation. In case of the magnitude of the tension was large, deformation of the cloth was observed. Conversely, when the magnitude of the pressure by the roller was small, a cloth slipped and could not be rotated properly.

Cloth has anisotropic mechanical characteristics, and those might affect so much the curvature of the sewing trajectory. Measurement was carried out to investigate the relationship between the difference of mechanical properties of a cloth in various directions and sewing trajectories. Here, we call the direction of a cloth "warp" when warp direction is the same as the direction of a line that connects the solenoid and the roller, and we call the direction of a cloth "bias" when bias direction of the cloth is the same as the direction 
of the line. We call it "warp start" that the cloth is directed to the "warp" direction or call it "bias start" if the cloth is directed to the "bias" direction when sewing starts. Experiments were carried out to investigate the difference of the sewing trajectories under the conditions of the "warp start" or the "bias start". The trajectories are shown in Figure 3a and 3b.

Taken in Figure 3a and 3b

It was revealed that in the both conditions of the "warp start" and the "bias start", a sewing trajectory was displaced to the inside of the desired trajectory when the cloth directed to the "bias" direction. Figure 4 shows the fluctuations of the radius of curvature of the sewing trajectory. It was confirmed that the curvature of the trajectory was not constant in both conditions of the "warp start" and the "bias start". The fluctuation was periodic and the phase was shifted according to the "warp start" or "bias start".

Taken in Figure 4

The reason of the fluctuation was the growth of a wrinkle on a cloth between two points, solenoid and roller as shown in Figure 5. The mechanical characteristics of a cloth are different according to directions. A cloth is much more easily stretched in bias direction than in other directions, and a wrinkle grew only when a cloth was directed to "bias", if the tension applied to a cloth between the two points was constant. It might affect the fluctuation of the radius of curvature of the sewing trajectory.

\section{Taken in Figure 5}

A simple measurement was carried out to estimate the magnitude of the force when the wrinkle grew. Five sample cloths were used for the measurement. A point on a cloth was fixed then a tension was applied at 
another point, and the tension was measured. Distance between two points was the same as the distance between the solenoid and the roller, the magnitude of the tension when displacement was $10 \mathrm{~mm}$ and $20 \mathrm{~mm}$ are shown in table I. As the tension was manually measured with a spring balancer, the results were not so precise, but the comparison between cloths might be possible. The wrinkle grew easily in order of the cloth No. 2, No. 1, No. 5, No. 3 and No. 4. From the comparison of the results and the properties measured with KES instruments, this deformation seemed to be most related with shearing property.

Taken in Table I

The growth of the wrinkle should be prevented because the wrinkle brought about a failure of sewing processes. To avoid the growth of the wrinkle, it was necessary to control the tension properly not to stretch the cloth in "bias" direction because it was the cause of inaccurate cloth guiding. To perform this control, relationship between the properties and the deformations of cloths had to be clear. Five cloth samples were prepared and the mechanical properties were measured with KES measurement instruments. Tensile, shear, bending, frictional force (with 50gf friction apparatus) and roughness of the surface of the five cloth samples were measured. The results of the measurement for each cloth are shown in Table II. No.2 and 5 samples were hardly stretched in the warp and bias directions, and No. 4 was easily stretched. The No 4 was hardly stretched in the weft direction and No. 1, 3 and 5 were easily stretched. Thick cloths such as No. 3 and 4 were hardly sheared in warp direction. Thin cloths such as No. 1 and 2 were easily sheared, and the cloth No. 5 was intermediate. These cloth samples had the same shearing characteristic in weft direction as in warp direction. No.1 to 3 were bent very easily, and No. 4 and 5 were relatively hard to bend in warp direction. These cloth samples had the same bending characteristic in weft direction as in warp except No.2 that was hardly bent. The frictional coefficient and the standard deviation of the roughness of the surface of 
each cloth were derived form the measurement of friction.

Taken in Table II

\section{A combined mechanism}

The growth of the wrinkle prevented to form fine stitch while sewing a cloth with a guiding mechanism consisted of a solenoid and a roller. To avoid the deformation of a cloth, it was necessary to decrease a tension applied to a cloth in "bias" direction. On the other hand, when the tension was small in every direction of the cloth, the correction of the deformation around the holding point could not be performed in "warp" or "weft" direction. The tension between the holding point of the cloth and the roller had to be controlled properly according to the direction of a cloth while sewing. To control this tension, it was required to add some controllable vertical sliding mechanism to the roller. A DC solenoid was utilized to control the roller pressure. The pressure of an iron core of a solenoid could be controlled regulating the magnitude of the voltage applied to the solenoid. The roller was connected at one end of the solenoid and a shaft was put through a linear bush prepared beside the solenoid to fix the revolving direction of the roller. The weight of the roller part was applied to the solenoid. The returning spring of the solenoid was changed to stiffer one so that the roller part did not drop when the solenoid was not applied a voltage. As the frictional coefficient was changed according to the pressure of the solenoid, it was necessary to determine the relationship between the applied voltage and the tension that the roller with solenoid exerted on a cloth. The force of the iron core pushing out from the solenoid could be adjusted by the voltage depending on the direction of a cloth. The same control system was utilized for this mechanism as the one used for the solenoid to hold a cloth. Figure 6 shows the appearance of the device. 
Taken in Figure 6

The relationship between the tension on a cloth and the applied voltage to the roller of the combined mechanism was observed. The tension was measured three times and averaged as a result as shown in Figure 7. Hysteresis was observed and this should be taken into account to control the mechanism. The sewing experiments were performed with this combined guiding mechanism. Each experiment was started from the "warp start" condition. The point where the solenoid was holding a cloth was located as the same position 70mm from the sewing needle as before. The solenoid of 3mm stroke and the maximum load of about 58gf per $10 \mathrm{~mm}$ of roller width connected to the roller. This maximum load was strong enough to avoid slack of the cloth, but in the "bias" direction, the tension caused by the load of the solenoid connected to the roller was so strong that a wrinkle grew. To avoid the growth of a wrinkle, the voltage applied to the solenoid was controlled on the basis of the mechanical properties and the direction of the cloth. The load for the optimal trajectory was obtained for each cloth, with the experiments varying the voltage applied to the solenoid. The timing of the increase or decrease of the voltage was symmetry about the time when the cloth is in "bias" direction. The value and the timing of the voltage change was different owing to the mechanical properties of the cloth. Utilizing the relationship between the voltage and the load, the relationship of the direction of cloth versus the load was derived. The comparison of the radius of curvature is shown in Figure 8 in the two conditions that the voltage applied to the solenoid was controlled and not controlled. It is shown in the graph that the fluctuation of the curvature was smaller in the case with control compared to the case without control. The order of the magnitude of the load to keep the proper tension of the cloth was the sample No.2, 1, 5, 3 and 4 from the smallest one, and the correlation coefficient between the load and the shear coefficient of the cloth was 0.98 . About the sample No.1 and 2, the coefficient of shear did not differ 
so much but a great difference was observed in the results of the experiments. The friction between the cloth and the roller was examined, as the properties such as surface friction might be related to this difference. In the measurements, the roller revolving at a constant speed was pressed on each cloth to examine the friction. The cloth was pulled to the contrary direction to the revolution of the roller with a spring balancer, to keep the cloth halt. The tension was measured when the movement of the cloth was balanced. The approximate relationship shown in Table III was obtained as the result of the measurement. The surface friction of the cloth No.2 was greater than any other samples. From the results of the measurements with KES instruments, the frictional coefficient and the degree of the surface unevenness of the cloth No.2 was the smallest among the samples. As the surface unevenness of the cloth No.2 was small, the area contacting with the roller surface was large, and the value of the frictional coefficient might become bigger than that of the cloth No.1. This was thought to be a reason that the less vertical load was required for the cloth No.2 than the cloth No.1 in the bias direction. When the direction of the roller was in the "bias" direction of a cloth, the magnitude of the tension should be as small as the cloth did not slacken. The shear and the surface properties of the cloth were thought to be much related to the wrinkle which grew when the tension between the solenoid and the roller was large. To control the load applied to a cloth, shear property, surface unevenness and surface frictional force should be taken into consideration. The load of the roller connected to the solenoid should be decreased as the shearing stiffness was smaller and the surface of cloth was smoother.

\section{Taken in Figure 7}

Taken in Figure 8

Taken in Table III 
Conclusion

We developed a guiding mechanism for processes of curved stitch. The mechanism was consisted of a solenoid and a roller. In the sewing process, a cloth was fed by a sewing machine, and the solenoid held a point on a cloth to form curved stitch. A roller was utilized to avoid deformations when the stitches were formed. The sewing trajectory was distorted for some cloths because of deformations. It was confirmed that the deformations were owing to the mechanical properties of a cloth. We succeeded to depress the deformations and obtained correct trajectory by the control of the pressure of the rollers. 
References

Carvalho, M. and Ferreira, F. N. (2003) “A step on automatic sewability evaluation and seam control on an overlock sewing machine”, International Conference on Mechatronics, ICOM 2003, pp. 563-568.

Rocha, A. M., Lima, M. F., Ferreira, F. N. and Araujo, M. D. (1996) "Development in automatic control of sewing parameters”, Textile Research Journal, Vol. 66, No. 4, pp. 251-256.

Rogale, D. and Dragcevic, Z. (1998) "Portable computer measuring systems for automatic process parameter acquisition in garment sewing processes”, International Journal of clothing science and technology, Vol. 10, No. 3-4, pp. 283-292.

Stylios, G and Sotomi, O. J. (1994) “Neuro-fuzzy control system for intelligent sewing machines”, IEE Conference Publication, Issue 395, pp. 241-246. 
Legends

Figure 1. Appearance of the cloth guiding mechanism

Figure 2. Arrangement of a sewing needle, a solenoid and a roller

Figure 3a. Sewing trajectories of warp start

Figure 3b. Sewing trajectories of bias start

Figure 4. Fluctuations of the radius of curvature of sewing trajectories

Figure 5. Wrinkle observed when tension was applied to a fabric

Figure 6. Appearance of the device

Figure 7. Relationship between a tension on a cloth and an applied voltage to the roller

Figure 8. Comparison of the radius of curvature

Table I. Magnitude of the tension applied to a fabric in bias direction

Table II. Mechanical properties of sample fabrics

Table III. The relationship between the pressure applied to roller and the tension applied to the fabric 


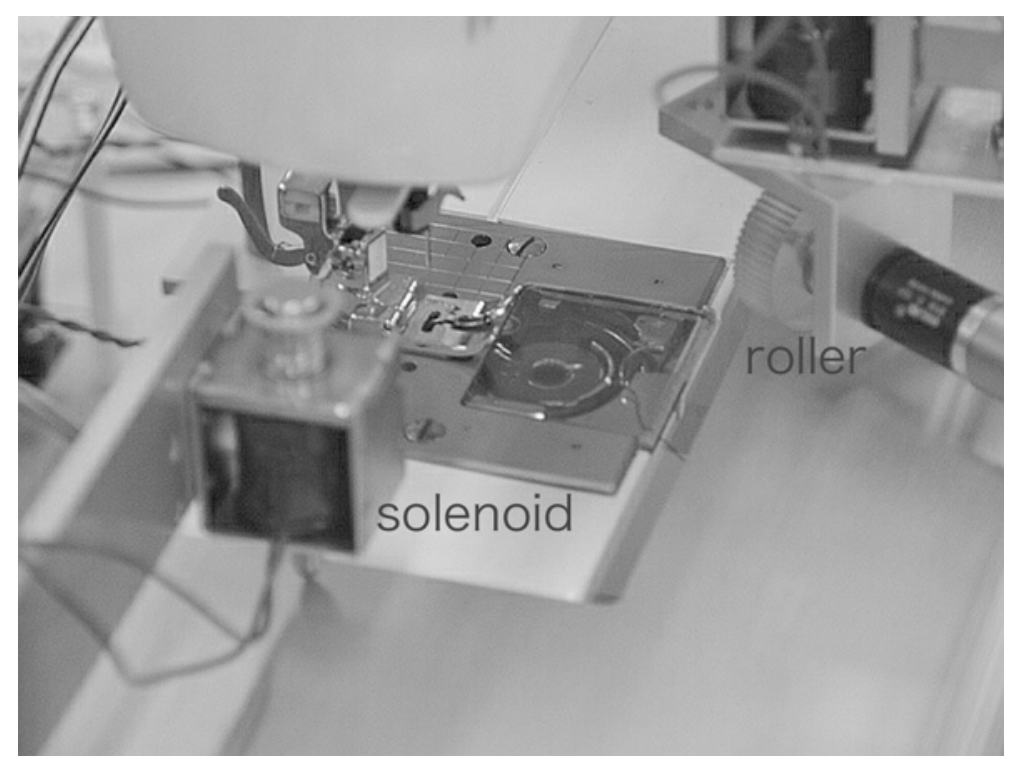

Figure 1. 


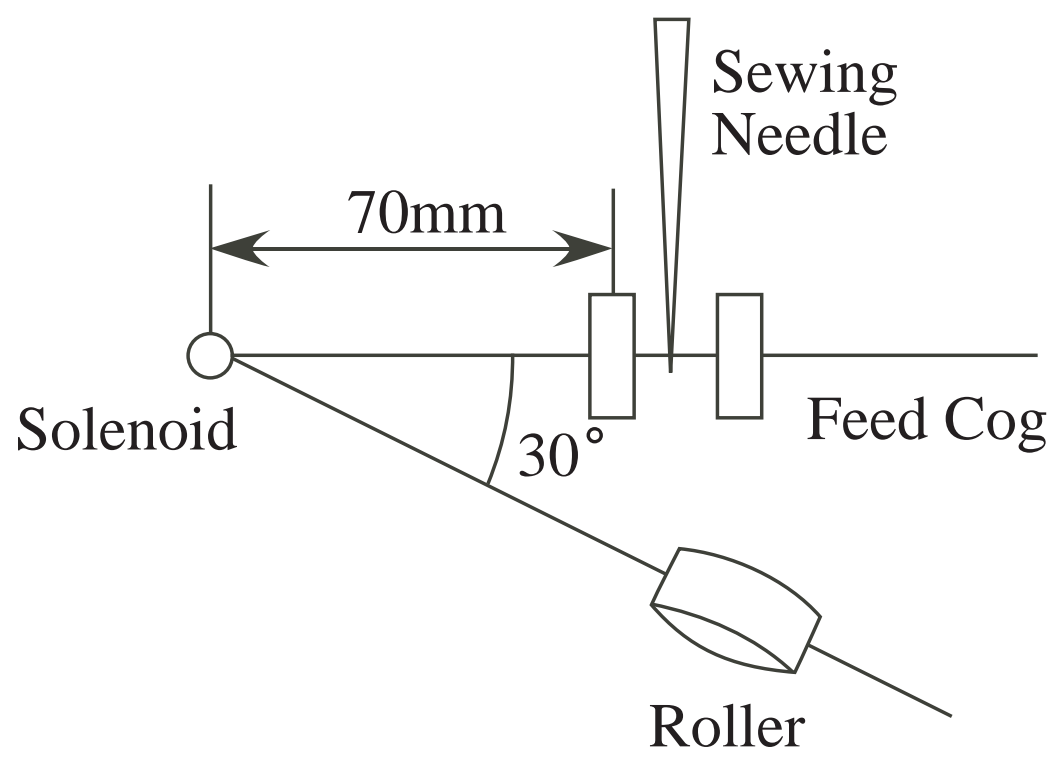

Figure 2. 


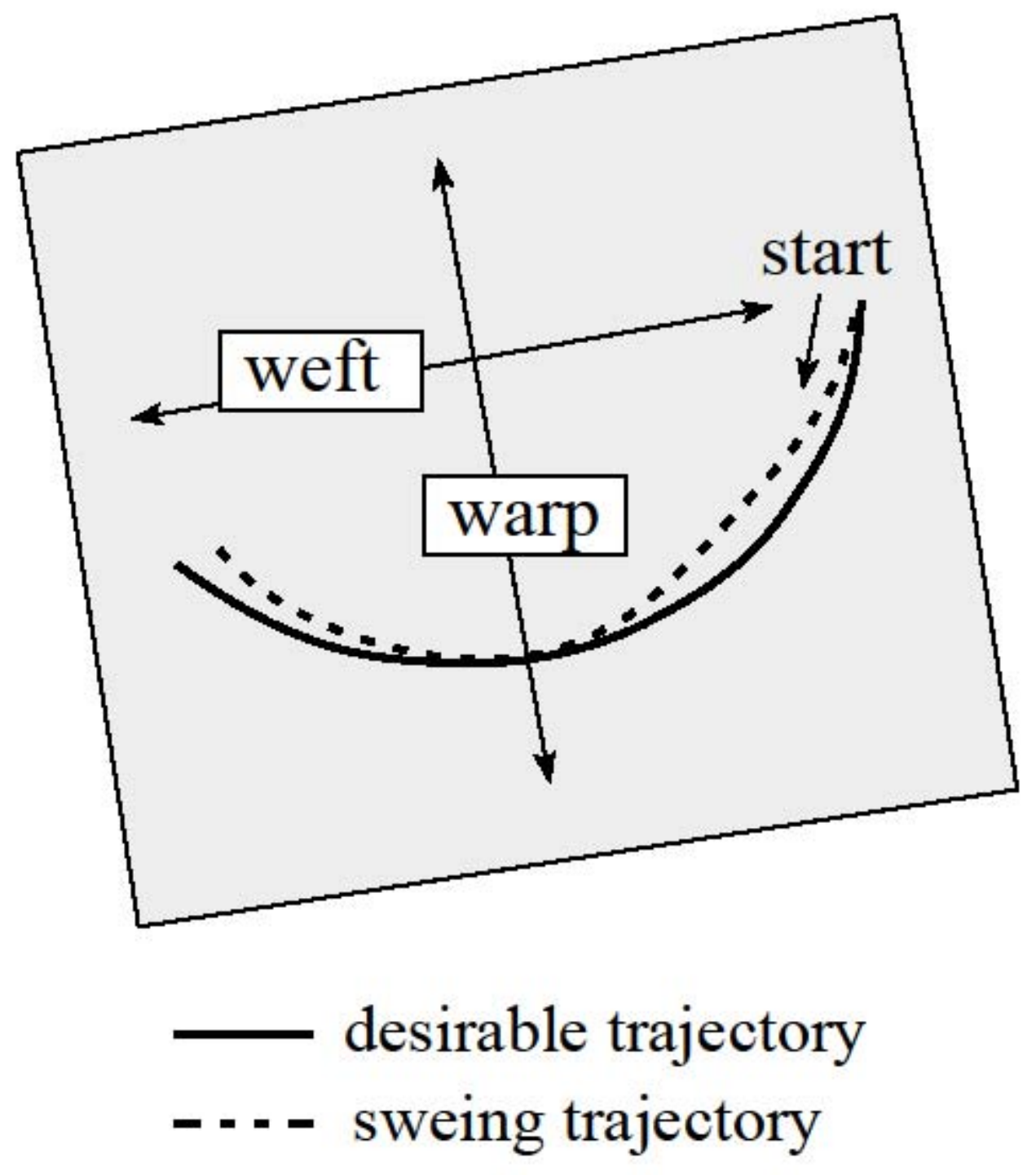

Figure 3a. 


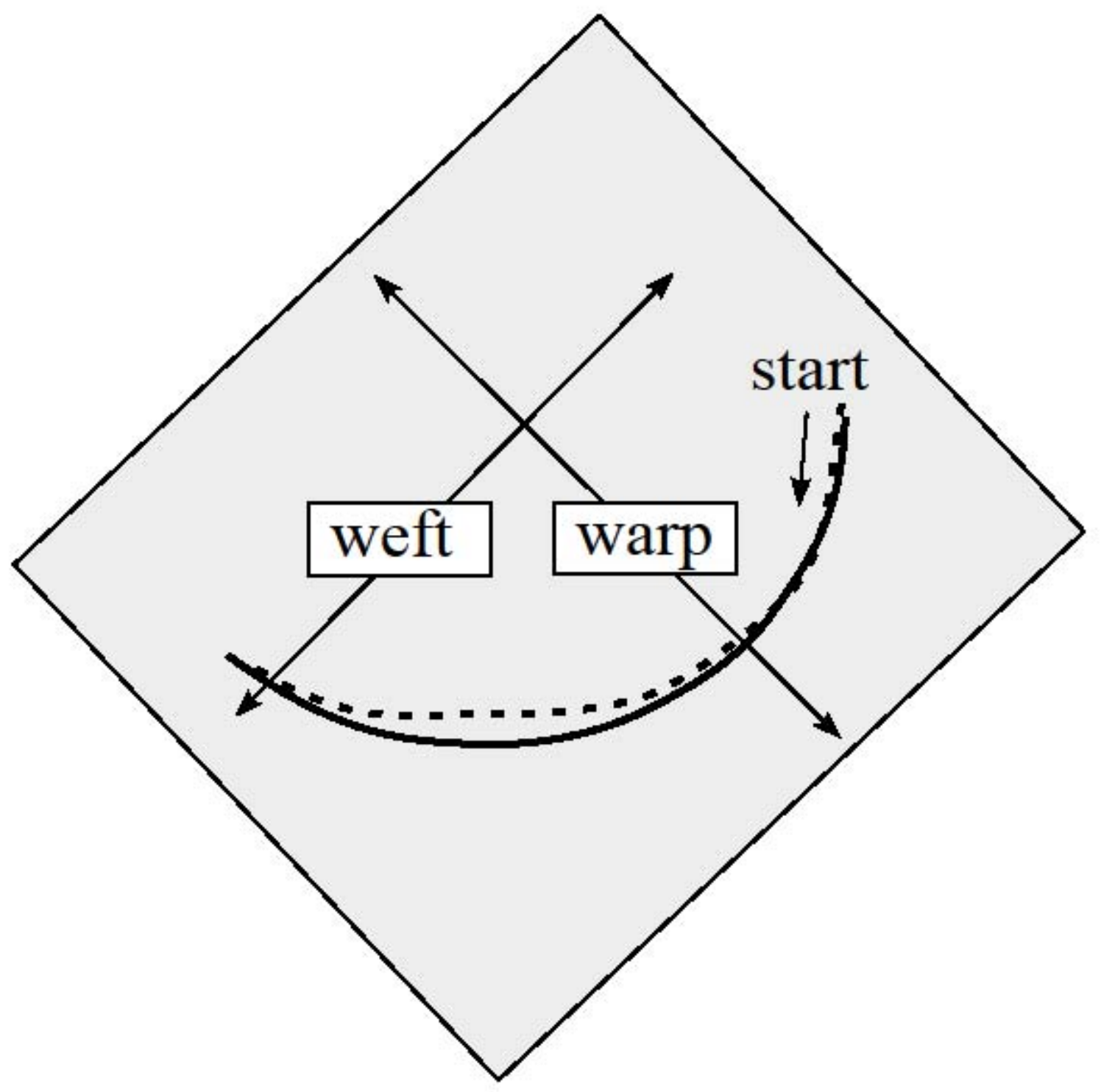

Figure 3b. 


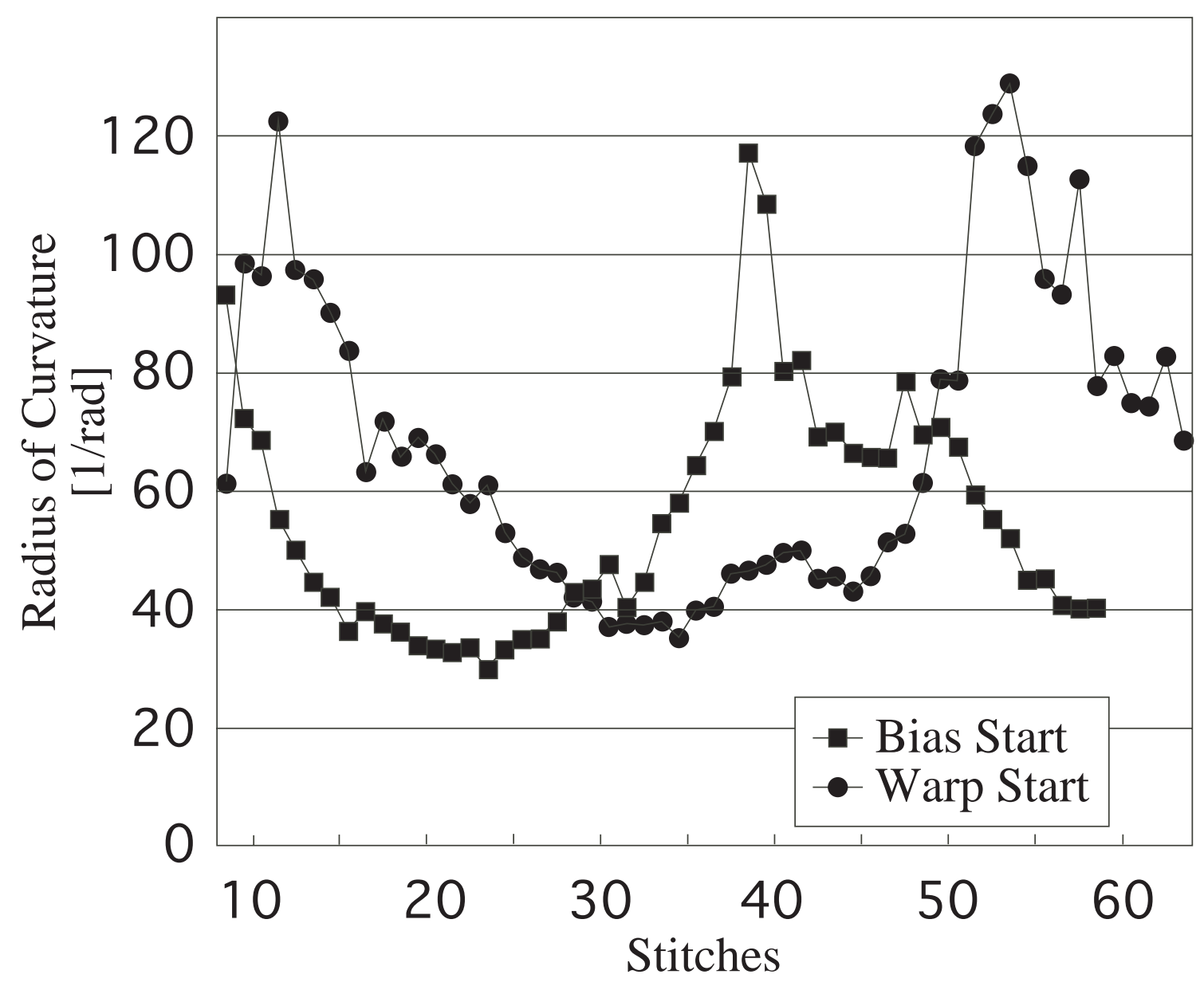

Figure 4 . 


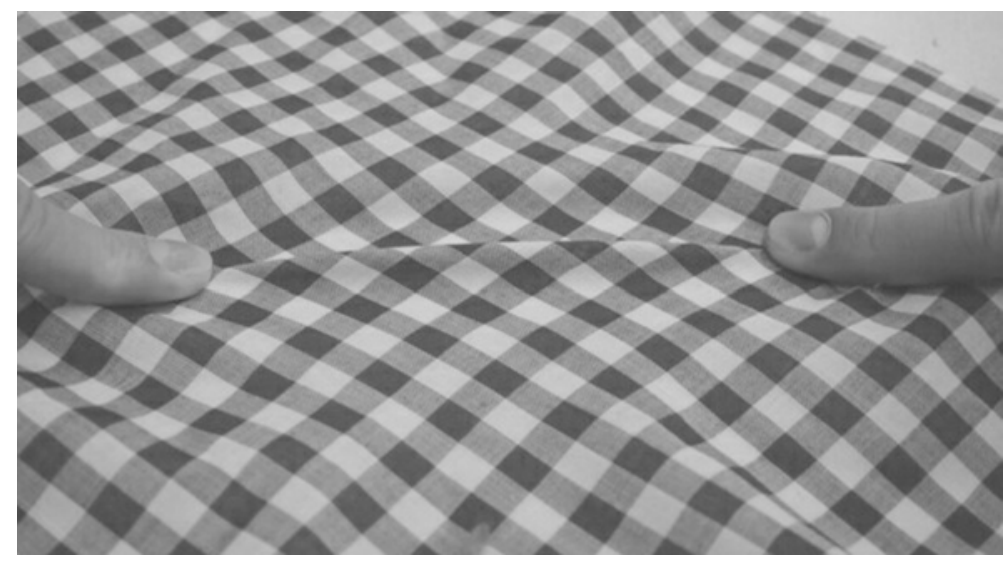

Figure 5. 


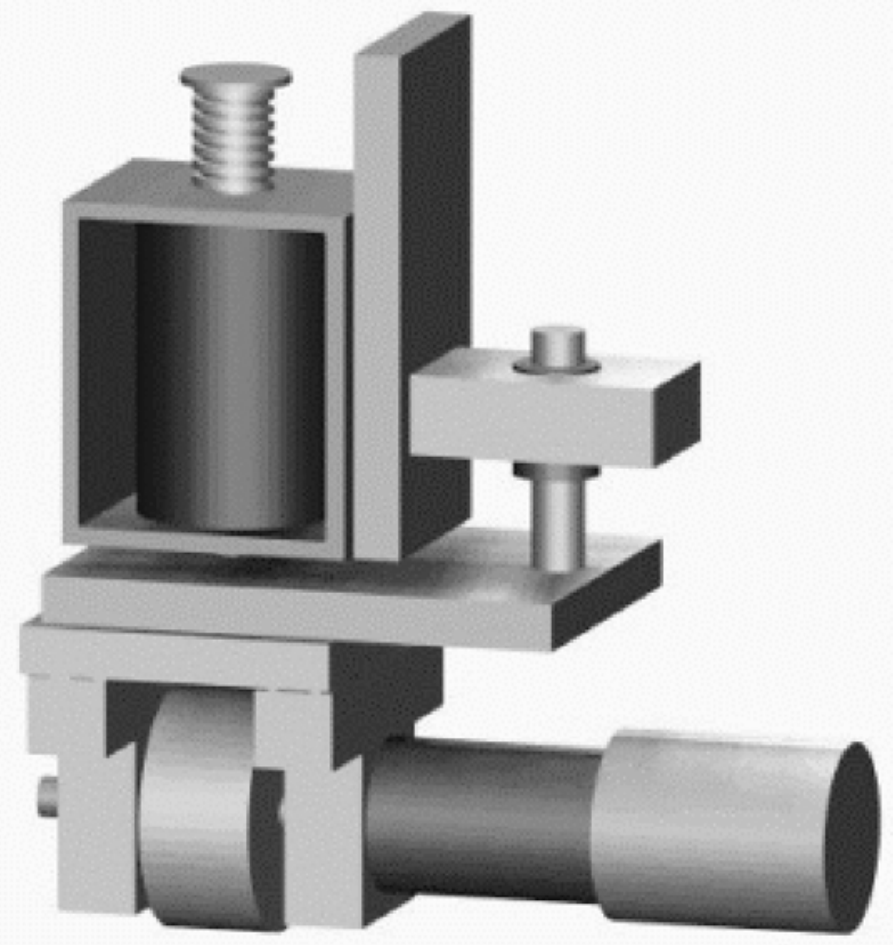

Figure 6.

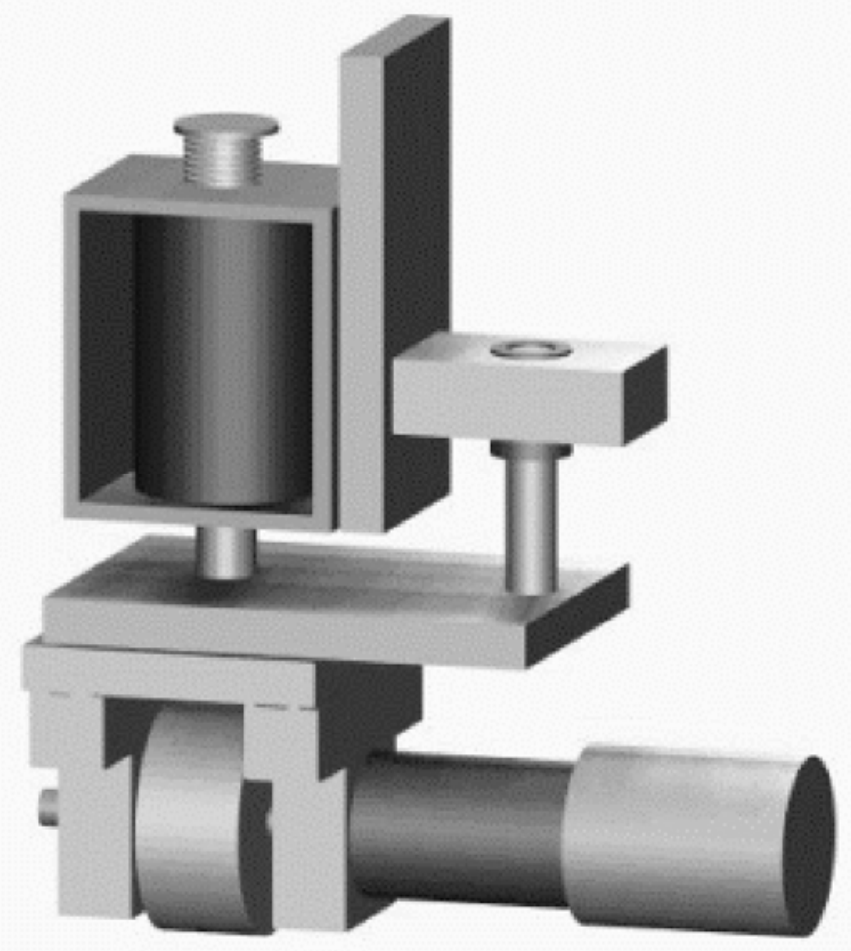

Figure 6b. 


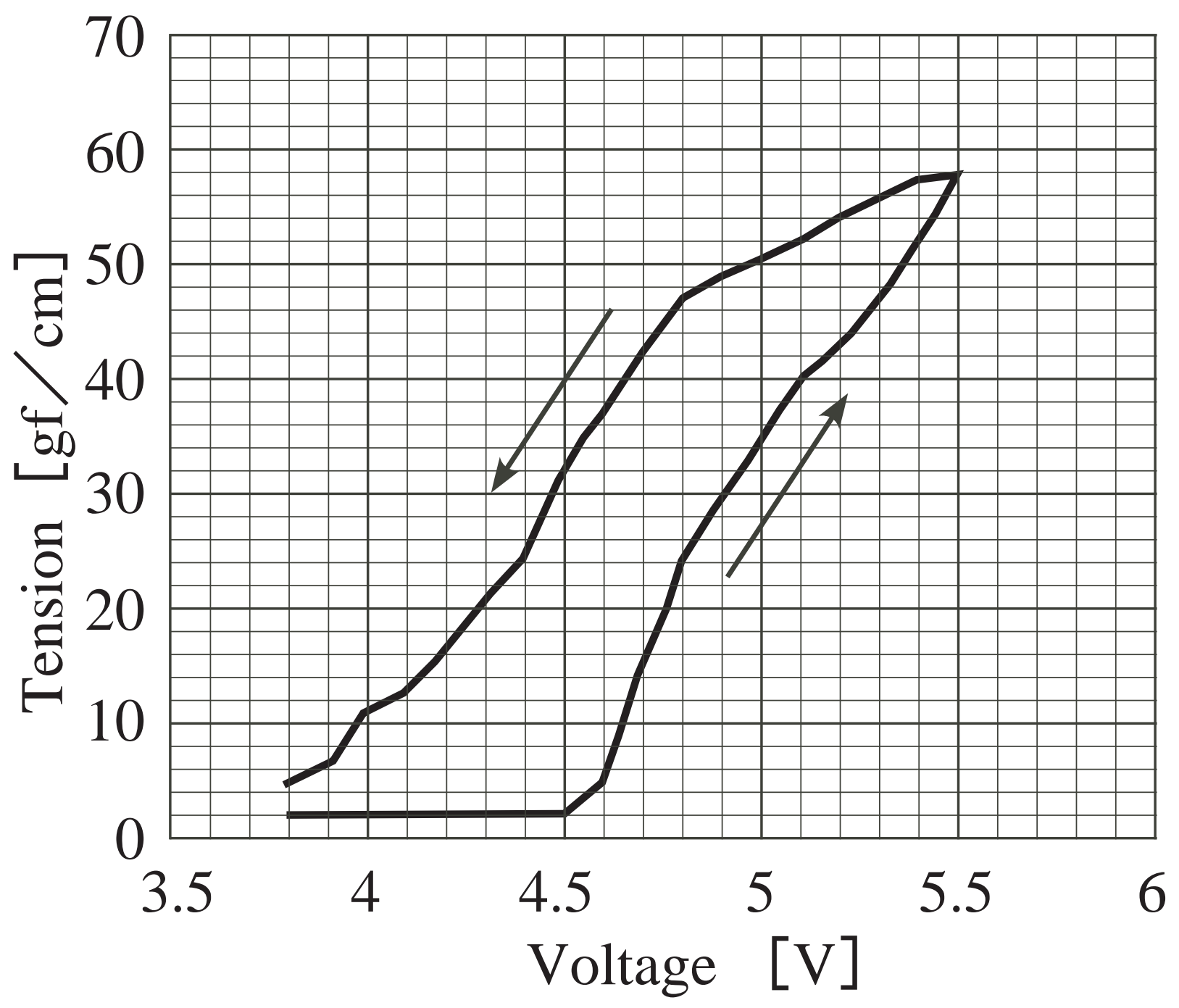

Figure 7. 


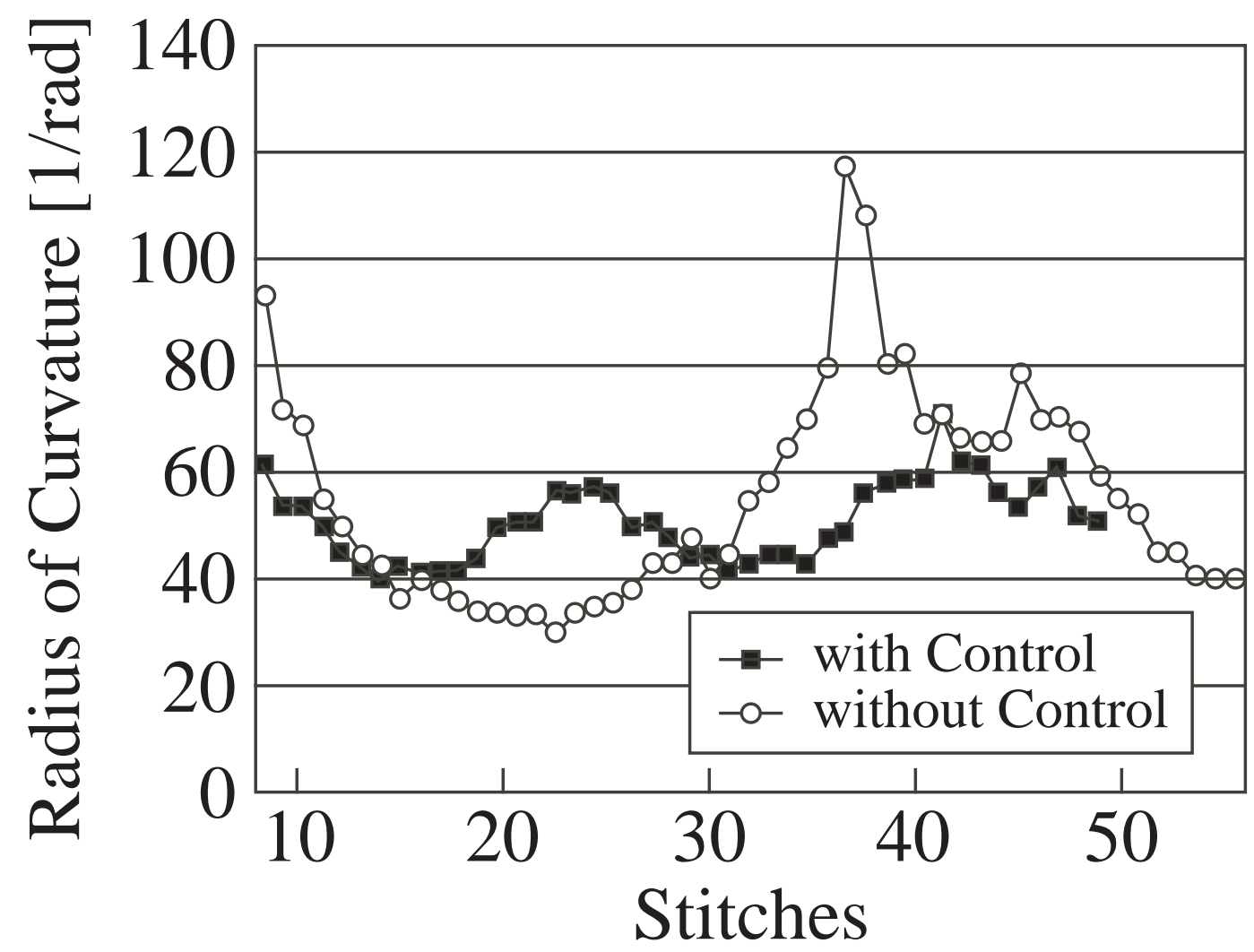

Figure 8. 
Table I. Magnitude of the tension applied to a fabric in bias direction cloth No. 122 3 extended length $10 \mathrm{~mm} \quad 15 \mathrm{gf} \quad 10 \mathrm{gf} 110 \mathrm{gf} 130 \mathrm{gf} \quad 30 \mathrm{gf}$ extended length $20 \mathrm{~mm} \quad 70 \mathrm{gf} \quad 60 \mathrm{gf} 300 \mathrm{gf} 350 \mathrm{gf} 120 \mathrm{gf}$ 
Table II. Mechanical properties of sample fabrics

\begin{tabular}{|c|c|c|c|c|c|c|}
\hline & 1 & 2 & 3 & 4 & 5 \\
\hline & & 3.68 & 4.38 & 7.77 & 12.68 & 3.9 \\
\hline \multicolumn{2}{|l|}{\begin{tabular}{|l|} 
weight $[\mathrm{g}]$ \\
thickness $[\mathrm{mm}]$
\end{tabular}} & 0.18 & 0.14 & 0.4 & 0.66 & 0.16 \\
\hline \multicolumn{2}{|l|}{ density $\left[\mathrm{g} / \mathrm{cm}^{3}\right]$} & 0.511 & 0.782 & 0.486 & 0.48 & 0.609 \\
\hline \multirow{4}{*}{ extention warp } & EMT [\%] & 4.83 & 1.16 & 3.78 & 7.47 & 1.83 \\
\hline & LT [-] & 0.671 & 0.767 & 0.72 & 0.696 & 0.809 \\
\hline & WT $\left[\mathrm{gf} / \mathrm{cm}^{2}\right]$ & 8.1 & 2.8 & 6.8 & 13 & 3.7 \\
\hline & RT [\%] & 56.8 & 73.2 & 55.2 & 47.7 & 71.6 \\
\hline \multirow{4}{*}{ extention weft } & EMT [\%] & 7.98 & 0.98 & 6.32 & 4.68 & 3.22 \\
\hline & \begin{tabular}{|l|l} 
LT [-] \\
\end{tabular} & 0.654 & 0.898 & 0.756 & 0.748 & 0.807 \\
\hline & WT $\left[\mathrm{gf} / \mathrm{cm}^{2}\right]$ & 13.1 & 2.2 & 12 & 8.8 & 6.5 \\
\hline & RT [\%] & 51.3 & 88.6 & 50.6 & 56 & 66.2 \\
\hline \multirow{4}{*}{ extention bias } & EMT [\%] & 9.35 & 1.46 & 6.76 & 8.13 & 2.73 \\
\hline & LT [-] & 0.618 & 0.863 & 0.695 & 0.701 & 0.835 \\
\hline & WT $\left[\mathrm{gf} / \mathrm{cm}^{2}\right]$ & 14.5 & 3.2 & 11.8 & 14.3 & 5.7 \\
\hline & RT [\%] & 45 & 71.4 & 46.4 & 51.2 & 71.1 \\
\hline \multirow{3}{*}{ shear warp } & $G$ & 0.88 & 0.74 & 2.08 & 2.48 & 1.52 \\
\hline & $2 \mathrm{HG}$ & 2.5 & 0.98 & 4.45 & 5.83 & 3.13 \\
\hline & $2 \mathrm{HG} 5$ & 3.38 & 3.13 & 8.28 & 7.83 & 6.5 \\
\hline \multirow{3}{*}{ shear weft } & $G$ & 0.93 & 0.78 & 2.17 & 2.92 & 1.39 \\
\hline & $2 \mathrm{HG}$ & 2.48 & 0.8 & 4.23 & 5.7 & 2.35 \\
\hline & $2 \mathrm{HG} 5$ & 3.63 & 2.93 & 8.53 & 9.1 & 5.83 \\
\hline \multirow{2}{*}{$\begin{array}{l}\text { bending } \\
\text { warp }\end{array}$} & $\mathrm{B}$ & 0.058 & 0.104 & 0.203 & 0.358 & 0.0832 \\
\hline & $2 \mathrm{HB}$ & 0.058 & 0.095 & 0.28 & 0.426 & 0.105 \\
\hline \multirow{2}{*}{$\begin{array}{c}\text { bending } \\
\text { weft }\end{array}$} & $\mathrm{B}$ & 0.038 & 0.276 & 0.118 & 0.252 & 0.038 \\
\hline & $2 \mathrm{HB}$ & 0.027 & 0.164 & 0.121 & 0.286 & 0.04 \\
\hline \multirow{2}{*}{ surface } & \begin{tabular}{|l|} 
frictional \\
coefficient
\end{tabular} & 0.043 & 0.034 & 0.04 & 0.044 & 0.039 \\
\hline & $\begin{array}{l}\text { standard } \\
\text { deviation }\end{array}$ & 3.49 & 0.41 & 1.17 & 5.35 & 2.25 \\
\hline
\end{tabular}


Table III. The relationship between the pressure applied to roller and the tension applied to the fabric

\begin{tabular}{|c|c|c|c|c|c|}
\hline \multirow{6}{*}{$\begin{array}{l}\text { Load } \mathrm{gf} / \mathrm{cm} \\
\text { tension } \mathrm{gf}\end{array}$} & & 60 & 40 & 20 & 10 \\
\hline & & 120 & 50 & 10 & 5 \\
\hline & 2 & 160 & 80 & 20 & 10 \\
\hline & 3 & 130 & 60 & 20 & 5 \\
\hline & 4 & 120 & 60 & 20 & 10 \\
\hline & 5 & 120 & 50 & 20 & 10 \\
\hline
\end{tabular}

